Оригинальные статьи / Original articles

https://doi.org/10.18619/2072-9146-2020-6-99-103 уДК 635.1/.7:663.15

Ж.А. Семенова ${ }^{1}$, Н.Е. Посокина', В.И. Терешонок ${ }^{2}$

${ }^{1}$ Всероссийский научно-исследовательский институт технологии консервирования - филиал Федерального государственного бюджетного научного учреждения «Федеральный научный центр пищевых систем им. В.М. Горбатова» РАН (ВНИИТеК - филиал ФГБНУ «ФНЦ пищевых систем им. В.М. Горбатова» РАН)

142703, Россия, Московская область,

г. Видное, ул. Школьная, д.78

www.vniitek.ru, vnikopltok@yandex.ru

${ }^{2}$ Федеральное государственное бюджетное научное учреждение "Федеральный научный центр овощеводства" (ФГБНУ ФНЦО)

143072, Россия, Московская область, Одинцовский район, п. ВНИИССОК, ул. Селекционная, д. 14

Конфликт интересов: Авторы заявляют об отсутствии конфликта интересов.

Для цитирования: Семенова Ж.А., Посокина Н.Е., Терешонок В.И. Влияние углеводной корректировки сырья на рост молочнокислых микроорганизмов в процессе направленного ферментирования овощей. Овощи России 2020;(6):99-103. https://doi.org/10.18619/20729146-2020-6-99-103

Поступила в редакцию: 23.07.2020

Принята к печати: 06.11.2020

Опубликована: 20.12.2020

Jeanne A. Semenova',

Nataliya E. Posokina ${ }^{1}$,

Vladimir I. Tereshonok ${ }^{2}$

Russian Research Institute of Canning Technology - Branch of V.M. Gorbatov Federal Research Center for Food Systems of RAS (VNIITeK - Branch of Gorbatov Research Center for Food Systems) 78, Shkolnaya Street, Vidnoe,

Moscow region, 142703, Russia

www.vniitek.ru, vnikopltok@yandex.ru

${ }^{2}$ Federal State Budgetary Scientific Institution Federal Scientific Vegetable Center (FSBSI FSVC) 14, Selectsionnaya str., VNIISSOK, Odintsovo district, Moscow region, Russia, 143072

Conflict of interest. The authors declare no conflict of interest.

For citations: Semenova Je.A., Posokina N.E., Tereshonok V.I. Influence of carbohydrate correction of raw materials on the growth of lactic acid microorganisms in the process of directed fermentation of vegetables. Vegetable crops of Russia. 2020:(6):99-103. (In Russ.)

https://doi.org/10.18619/2072-9146-2020-6-99-103

Received: 23.07.2020

Accepted for publication: 06.11.2020

Accepted: 20.12.2020
Влияние углеводной

корректировки сырья

на рост молочнокислых

микроорганизмов

в процессе направленного

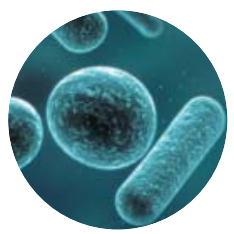

Резюме

Актуальность. Использование определенных одиночных или смешанных культур молочнокислых микроорганизмов и обязательный контроль процесса ферментации белокочанной капусты имеют большое значение для обеспечения получения продукта стабильного качества без необходимости использования каких-либо химических консервантов или жестких условий обработки. Важную роль в данном процессе играют содержащиеся в сырье углеводы, поскольку именно углеводы являются основным источником поддержания жизнеспособности молочнокислых микроорганизмов. Корректируя углеводную составляющую сырья на разных этапах ферментации, возможно добиться поддержания высокого титра микроорганизмов, получая при этом не только качественный продукт, но и придавая ему наряду с пребиотическими (источник клетчатки) и пробиотические свойства.

Материал и методика. Целью данной исследовательской работы было обеспечение направления хода биотехнологического процесса в сторону стабилизации концентрации функциональной микрофлоры (стартовых систем молочнокислых микроорганизмов), под действием вносимых углеводных питательных веществ. В работе изучались две заквасочные системы микроорганизмов, являющиеся основополагающими микроорганизмами при квашении капусты, а именно системы, состоящие из предферментационной культуры Leuconostoc mesenteroides и одной из культур лактобактерий: Lactobacillus plantarum или Lactobacillus brevis. Для исследования использовали микробиологический метод определения количества микроорганизмов путем посева в агаризованную питательную среду, а также обработка экспериментальных данных.

Результаты. Установлено, что внесение углеводной добавки позволяет стабилизировать динамику изменения концентрации изученных стартовых систем на одном и том же начальном уровне на всем протяжении основной стадии ферментации.

Ключевые слова: стартовые системы, белокочанная капуста, ферментация, углеводная добавка, концентрация микроорганизмов, Leuconostoc mesenteroides, Lactobacillus plantarum, Lactobacillus brevis

\section{Influence of carbohydrate correction of raw materials on the growth of lactic acid microorganisms in the process of directed fermentation of vegetables}

\section{Abstract}

Relevance. The use of certain single or mixed cultures of lactic acid microorganisms and the mandatory control of the fermentation process of white cabbage are of great importance to ensure that the product is of stable quality without the need for any chemical preservatives or harsh processing conditions. Carbohydrates contained in raw materials play an important role in this process, since carbohydrates are the main source of maintaining the viability of lactic acid microorganisms. By adjusting the carbohydrate component of raw materials at different stages of fermentation, it is possible to maintain a high titer of microorganisms, while obtaining not only a high-quality product, but also giving it, along with prebiotic (a source of fiber) and probiotic properties.

Materials and methods. The purpose of this research work was to ensure the direction of the biotechnological process in the direction of stabilization of the concentration of functional microflora (starting systems of lactic acid microorganisms), under the influence of introduced carbohydrate nutrients. In this work, we studied two fermentation systems of microorganisms that are fundamental microorganisms in the fermentation of cabbage, namely, systems consisting of a pre-fermentation culture of Leuconostoc mesenteroides and one of the cultures of lactobacilli: Lactobacillus plantarum or Lactobacillus brevis. The study used the usual microbiological method for determining the number of microorganisms by seeding in an agarized culture medium, as well as processing experimental data.

Results. As a result, we found that the introduction of a carbohydrate Supplement allows us to stabilize the dynamics of changes in the concentration of the studied starting systems at the same initial level throughout the main stage of fermentation.

Keywords: starter systems, white cabbage, fermentation, carbohydrate additive, concentration of microorganisms, Leuconostoc mesenteroides, Lactobacillus plantarum, Lactobacillus brevis 
$\Pi^{2}$ роцесс урбанизации современного общества характеризуется не только позитивными, но и рядом негативных изменений в области экологии, структуры и качества питания. В данной ситуации интересно внимание к ферментированным продуктам. Пищевая ценность ферментированных продуктов уже давно признана. Такие продукты используются практически во всем мире. С помощью ферментации увеличивается срок годности продуктов. Ферментированные продукты - это продукты, наделенные еще и определенными функциональными свойствами $[1,2,3]$.

В качестве ферментационных субстратов могут выступать как сырье животного, так и растительного происхождения.

Интерес к технологии ферментирования обусловлен следующими аспектами: благодаря применению различных технологий ферментированные продукты могут иметь разнообразный вкус и структурные свойства [3]. Также нельзя не отметить, что качество производимого ферментированного продукта является очень важным. Но важно подчеркнуть, что особенно это актуально в случае порой нестандартных растительных субстратов, поэтому, чем стабильнее необходим конечный продукт, тем более наукоемкой должна быть технология ферментации.

Нельзя не отметить глубокое укоренение микробиологических знаний в области производства молочных продуктов [4] и большую научную и практическую школу.

Исследования и наработки в области управления процессами ферментации овощного сырья, конечно, тоже имеются. Так еще в 50-х годах прошлого столетия было доказано, что применение чистых культур при квашении овощей не только ускоряет кислотообразование, но придает молочнокислому брожению большую направленность и способствует повышению качества квашеных овощей. Однако уже в те годы задумались о недостатках технологии, которая совмещала процессы брожения и хранения, требующие различных условий для их проведения [5].

Существует биотехнологическое изобретение на использование заквасочного микроорганизма Lactobacillus brevis для квашения капусты, датируемое 1991 годом [6]. Есть в России работа по применению стартовых молочных культур L. plantarum для квашения капусты [7]. Согласно литературным данным описаны инновационные методы квашения капусты с применением чистых культур. В литературе описаны коммерческие культуры-закваски, которые состоят из бактерий, образующих молочную кислоту и ароматические вещества (то есть условно делятся на 2 категории - кислотообразующую и ароматобразующую), к бактериям, используемым при производстве сквашенных растительных продуктов, относятся Lactobacillus casei, Lactobacillus helveticus, Lactobacillus lactis, Lactobacillus bulgaricus, Streptococcus thermophilus, Leuconostoc dextranicum, выбор и состав используемых комбинаций определяется желаемыми свойствами и условиями ферментации [8]. Также известны работы по ведению процессов молочнокислого брожения овощей, в частности, квашения капусты с использованием чистых культур Lactobacillus plantarum, Lactobacillus casei, L.acidophilus [9], а также работы по применению промышленной закваски «Наринэ» для квашения капусты [10]. Хотя согласно авторам [7] у нас в стране в большинстве случаев при изготовлении квашеной капусты используют традиционный метод, при котором капуста сквашивается только за счет эпифитной микрофлоры сырья.

Если речь идет о получении стандартизированного сброженного продукта типа квашеной капусты, то процесс управления ферментацией (брожением) овощей - сложный вопрос. Сложность, во-первых, заключается в том, что исследования по изучению направленной ферментации овощей сталкиваются с рядом непростых особенностей: в первую очередь ферментация овощей - это процесс преемственный, т.е. сначала участвуют одни микроорганизмы, затем эстафетную палочку подхватывают другие виды микроорганизмов; во вторую очередь - сырье в случае производства квашеной капусты не пастеризуется. В случае применения заквасок - проблема в установлении преемственности микроорганизмов - это очень большая трудность [2].

Во-вторых, сложностью является и тот факт, что в ассоциациях заквасочных штаммов необходимо учитывать, что между ними могут быть симбиотические (стимуляция развития культур при их совместном культивировании), антагонистические (подавление развития одной из культур при их совместном культивировании) и метабиотические взаимоотношения [11].

В-третьих, сложность, как и в любом биотехнологическом процессе, заключается в множественности параметров. Так, например, если рассмотреть параметры, оказывающие влияние только лишь на рост микроорганизмов, являющихся неотъемлемой частью биотехнологической ферментации, то мы увидим большой спектр параметров, а именно рН, содержание влаги, окислительно-восстановительный потенциал, содержание питательных веществ, антимикробные компоненты, температура, присутствие и концентрация газов, присутствие и активность других микроорганизмов и др. [12]

В комплексной программе развития биотехнологий (технологий с использованием микроорганизмов для получения необходимых человеку веществ / продуктов), в Российской Федерации на период до 2020 года говорится о важности для экономики и о необходимости массового внедрения биотехнологий и биотехнологических продуктов при модернизации технологической базы современного промышленного производства [13].

Во ВНИИТеК - филиале ФГБНУ «ФНЦ пищевых систем им. В.М. Горбатова» РАН проводятся обширные работы по изучению биотехнологических основ микробной трансформации растительного сырья на примере ферментации белокочанной капусты.

Основное нарушение течения биотехнологического процесса состоит в подавлении жизнедеятельности функциональной микрофлоры на жизненном цикле ее развития, которое вызывается нехваткой питательных веществ и наличием контаминирующей популяции [14].

Таким образом, базовым аспектом безопасной ферментации является сохранение функциональной микрофлоры 
на активном этапе и недопущение развития микроорганизмов порчи и т.п.

Для обеспечения этого аспекта необходимо достаточное количество сахаров (глюкозы и фруктозы) в исходном сырье, 4\% и выше, что гарантирует накопление достаточного количества молочной кислоты и других органических кислот, что позволяет исключить развитие нежелательной микрофлоры (гнилостных и маслянокислых микроорганизмов) [15]. Как известно, кочаны капусты белокочанной содержат 7-8\% сухого вещества, 4-6\% сахаров, в среднем 30 мг/100 г аскорбиновой кислоты, основная часть сахаров - это моносахара: 3,23\% (от 1,28 до 5,69\%), на фруктозу приходится примерно 0,7\%, на глюкозу - 1\% [16].

Очевидно, что сахаров, присутствующих в капусте, достаточно для проведения классической ферментации.

Известно, в классическом периодическом культивировании, т.е. культивировании, в котором единожды вносится питательная среда и культура, меняется темп роста микроорганизмов, кривые роста культуры проходят классические фазы: лаг-фаза, фаза экспоненциального роста, фаза замедленного роста, стационарная фаза и фаза отмирания. Известно, что в таких различных стадиях развития культуры одновременно со скоростью изменяется и физиологическая активность микроорганизмов. Быстрорастущие клетки потребляют вещества и образуют продукты обмена гораздо быстрее, чем клетки, рост которых начал замедляться. Первые обладают меньшей устойчивостью к неблагоприятным внешним факторам [8]. При непрерывном культивировании, в котором используются непрерывно обновляемые питательные среды, можно задержать культуру на любой стадии развития [17,18,19].

Цель исследования: изучение влияния углеводной корректировки сырья на развитие молочнокислых микроорганизмов в процессе направленной ферментации капусты белокочанной сорта Парус.

\section{Материалы и методы}

Для проведения эксперимента были выбраны:

стерильная модифицированная модельная среда на основе белокочанной капусты сорта Парус (исходное сырье предоставлено ФГБНу «Федеральный научный центр овощеводства»);

две стартовые (заквасочные) системы: L. mesenteroides / L. plantarum и L. mesenteroides / L. brevis.

Leuconostoc mesenteroides, Lactobacillus plantarum являются одними из основных молочнокислых бактерий при производстве квашеной капусты, также известно, что $L$. brevis является микроорганизмом, принимающим активное участие при естественном квашении капусты $[3,1]$.

L. mesenteroides и L. brevis являются гетероферментативными микроорганизмами, L. plantarum является гомоферментативным микроорганизмом [12].

Были использованы следующие штаммы микроорганизмов: штамм Leuconostoc mesenteroides subsp. mesenteroides 37Y, Lactobacillus brevis штамм B-1309, Lactobacillus plantarum штамм 578/26.

Штамм Leuconostoc mesenteroides subsp. mesenteroides 37Y, номер в коллекции ВКПМ: В-8818, культурально-мор- фологические признаки: клетки - овальные кокки, колонии - мелкие белого цвета, округлой формы [20]. Штамм предоставлен Всероссийской Коллекцией Промышленных Микроорганизмов ФГУП ГосНИИГенетика.

Lactobacillus brevis штамм B-1309, культурально-морфологические особенности штамма: мелкие палочки с закругленными концами, размером 0,7-1,0х2,0-4,0 мкм, грамположительные, неспорообразующие, гетерофакультативный анаэроб, сбраживает лактозу, глюкозу, фруктозу и другие сахара [21]. Штамм предоставлен Всероссийским научноисследовательским институтом пищевой биотехнологии филиалом Федерального государственного бюджетного учреждения науки Федерального исследовательского центра питания, биотехнологии и безопасности пищи.

Lactobacillus plantarum штамм 578/26 (родословная: штамм получен из Lactobacillus plantarum BКM B-578), культурально-морфологические особенности штамма: неподвижные палочки размером 0,9-1,2х3-8 мкм, грамположительные неспорообразующие аэротолерантные, сбраживают фруктозу, глюкозу, лактозу и другие сахара, обладает способностью к синтезу бактериоцинов [22]. Штамм предоставлен Всероссийским научно-исследовательским институтом пищевой биотехнологии - филиалом Федерального государственного бюджетного учреждения науки Федерального исследовательского центра питания, биотехнологии и безопасности пищи.

Подготовку культур проводили следующим образом: культуры, находящиеся на хранении, пересевали в жидкую питательную среду MRS, термостатировали при температуре $30^{\circ} \mathrm{C}$ в течение 72 часов, определяли начальный титр суспензии микроорганизмов.

Приготовление модельной среды осуществляли следующим образом: в измельченную простерилизованную массу белокочанной капусты сорта «Парус» вносили $\mathrm{NaCl}$ из расчета 1,5\% и аскорбиновую кислоту из расчета 0,035\%.

Инокуляцию модельной среды для проведения предварительной стадии ферментации проводили культурой Leuconostoc mesenteroides в количестве 1,75 $\pm 0,05 \%$. Полученную массу перемешивали, помещали в специальную емкость с возможностью откачивания воздуха, откачивали воздух и ставили в термостат на $+24^{\circ} \mathrm{C}$ на трое суток.

Затем в случае опыта без углеводной добавки в предварительно проферментированную массу вносили инокулят в двух вариантах: Lactobacillus brevis, Lactobacillus plantarum.

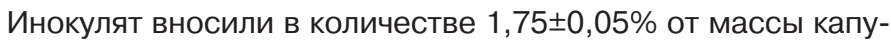
сты.

Для проведения опыта с углеводной добавкой был произведен расчет внесения этой углеводной добавки в массу, выполненный в предыдущих исследованиях. В предварительно проферментированную массу вносили 0,23\% глюкозы и 0,9\% фруктозы. Тщательно перемешивали. Далее вно-

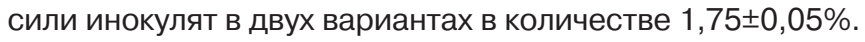

Отбирали на анализ, остальную массу расфасовывали по микроцентрифужным пробиркам типа «Эппендорф» и убирали обратно в термостат на $+24^{\circ} \mathrm{C}$. По прошествии семи суток после начала предварительной стадии, пробирки перемещали в холодильник для хранения при температуре $4 \pm 2{ }^{\circ} \mathrm{C}$. 
Отбор проб проводили на 0, 1, 4, 5, 7, 18, 29 сутки после начала основной стадии ферментирования.

Исследования проводили путем посева в агаризованную питательную среду МРС разведений отобранного образца, инкубирования посевов при температуре $30^{\circ} \mathrm{C}$ в течение 120 часов, подсчета всех видимых колоний и расчета количества микроорганизмов, присутствующих в 1 г пробы (концентрации). Подсчет количества микроорганизмов и доверительных интервалов производился по ГОСT ISO 72182015 [23].

\section{Результаты}

На рисунке 1 представлены данные по изменению концентрации молочнокислых микроорганизмов в модельной среде на основе капусты белокочанной сорта Парус без углеводной корректировки.

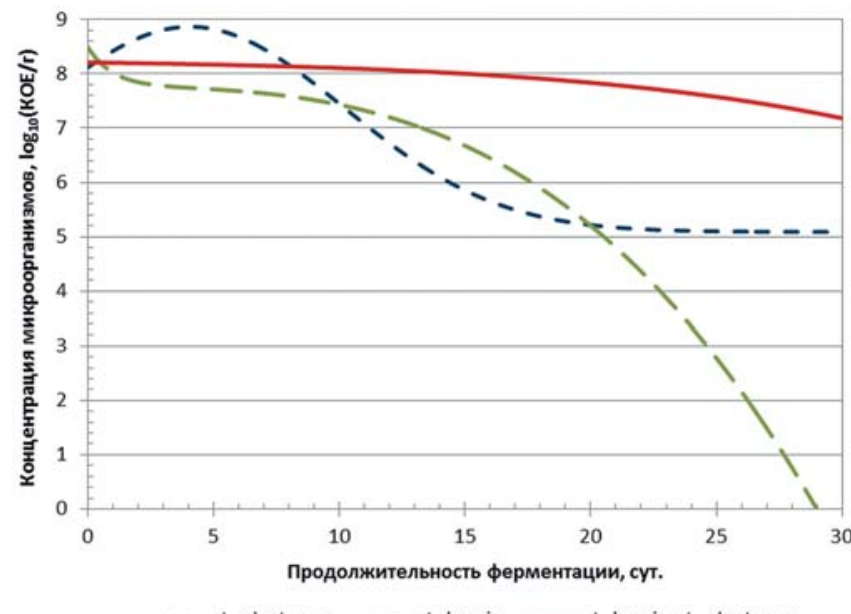

Рис. 1. Зависимость концентрации L plantarum, L. brevis и их консорциума от продолжительности основного этапа ферментации

Fig. 1. The dependence of the concentration of $L$ plantarum, $L$. brevis and consortium on the duration of the main fermentation stage

Анализ результатов экспериментальных данных и моделирования показал, что условия модельной среды, модифицированной на этапе предварительной ферментации, являются относительно неблагоприятными для развития микроорганизмов рода L. brevis, количество которых снижается с 8-го порядка до 0 за 28 суток ферментации. В то же время концентрация L plantarum снижается всего на 3 порядка за это же время.

Следует отметить, что динамика снижения количества молочнокислых микроорганизмов в консорциуме не так выражена, как у монокультур и снижение концентрации микроорганизмов за аналогичный период ферментации чуть больше одного порядка. Таким образом, мы видим явное преимущество использования молочнокислых микроорганизмов в качестве стартерных культур не в виде

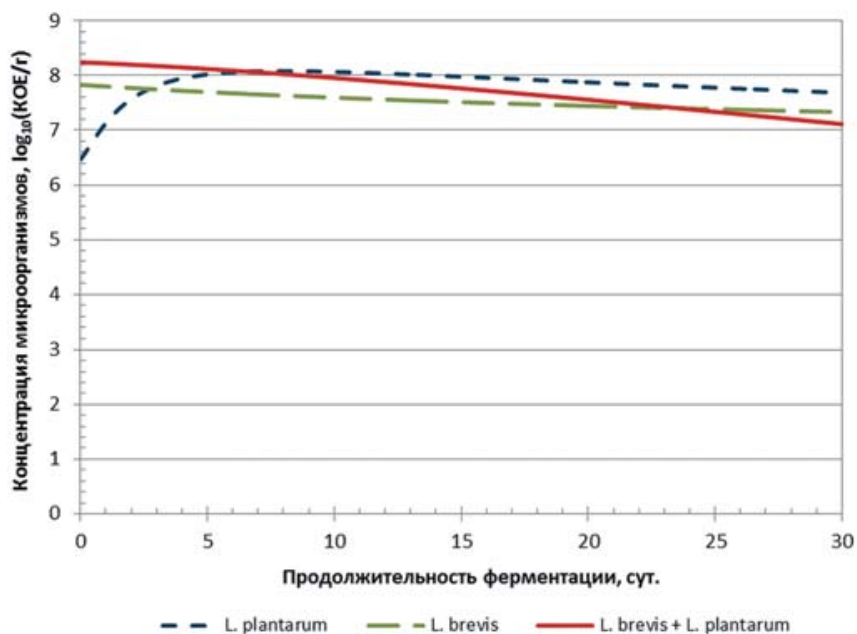

Рис. 2. Зависимость концентрации L. plantarum, L. brevis и их консорциума от продолжительности основного этапа ферментации после углеводной корректировки

Fig.2. The dependence of the concentration of $L$ plantarum, $L$. brevis and consortium on the duration of the main fermentation stage after carbohydrate adjustment

монокультур, а в виде консорциума этих микроорганизмов.

На рисунке 2 представлены данные по изменению концентрации молочнокислых микроорганизмов в модельной среде на основе белокочанной капусты сорта «Парус» с углеводной корректировкой среды. Углеводная корректировка была рассчитана на основании ранее проведенных исследований и составила 219 мг глюкозы на 100 г среды и 842 мг фруктозы соответственно.

После дополнительного внесения глюкозы и фруктозы в исследуемую среду, количество L. brevis снижается всего на 0,5 порядка за все время ферментации. У L. plantarum в первое время наблюдается значительный рост концентрации (практически на 2 порядка), который происходит в первые 7 сут ферментации, затем идет незначительное снижение, которое по прошествии 30 сут ферментации не достигает даже стартовой концентрации. В консорциуме наблюдается также незначительное снижение титра (с 8 до примерно 7 степени).

\section{Заключение}

Внесение углеводной добавки позволяет перевести ход ферментации с участием двух стартерных систем из хаотичного падения количества целевой микрофлоры в четко отрегулированный, направленный процесс. Данный прием привел к стабилизации процесса, как по значению уровня концентрации, так и по поддержанию этого уровня на протяжении всего периода основной стадии ферментации. 


\section{Об авторах:}

Жанна Александровна Семенова - научный сотрудник

Наталья Евгеньевна Посокина - кандидат технических наук, зав. лабораторией технологии консервирования, https://orcid.org/0000-0002-7857-6785 Владимир Ильич Терешонок - старший научный сотрудник лабораторноаналитического центра, кандидат с.-х. наук

\section{- Литература}

1. Позднякова О.Г., Казакова М.А., Австриевских А.Н., Позняковский В.М Микронутриентная поддержка молодого организма в условиях урбанизации современного общества. АПК России. 2019;26(3):436-441. Челябинск, ISSN 2587-8824.

2. Hutkins R.W. Microbiology and Technology of Fermented Foods. Blackwel Publishing. 2006

3. Шишлова Е.С., Посокина Н.Е., Лялина О.Ю. Основы ферментирования белоко чанной капусты. Вестник ВГУИТ. 2018;80(2):242-248. doi:10.20914/2310-1202 2018-2-242-248

4. Ганина В.И., Королева Н.С., Фильчакова С.А. Техническая микробиология продуктов животного происхождения: Учебное пособие. М.: ДеЛи принт, 2008. 352 с. 5. Выщепан А.Г., Мельман М.Е. Физико-химические основы соления и квашения овощей. Москва: Госторгиздат, 1952. 158

6. Турсунбаева Г.Н., Касенова А.А., Чуканов Н.К., Мукашева Г.М., Мурзашева Н., Рахимберлина P.M. Штамм бактерий Lactobacillus brevis, используемый при квашении капусты. Тип: авторское свидетельство. Номер свидетельства: SU 162239 А1 Патентное ведомство: СССР, 1991. Номер заявки: 4474295. Дата регистрации: 17.08.1988. Дата публикации: 23.01.1991. Заявитель: Институт Микробиологии и Вирусологии АН КАЗССР (Электронный ресурс: Elibrary: дата доступа 08042020 ) 7. Шлыкова А.Н., Устимова Е.А., Белокурова Е.С. Использование стартовых культур микроорганизмов Lactobacillus plantarum 8p-a3 и Lactobacillus fermentum 39 производстве квашеной капусты. Сборник трудов конференции Неделя науки СПБПУ Санкт-Петербург, 19-24 ноября 2018. С.47-50.

8. Белокурова Е.С., Иванченко О.Б. Биотехнология продуктов растительного происхождения: учебное пособие. СПб.: Издательство «Лань», 2019. 232 с. (Учебники для ВУЗов. Специальная литература

9. Павловская Л.М., Голубева С.Н. Перспективные направления научных исследований процессов консервирования овощей и фруктов: производство ферментированных продуктов. РУП «Научно-практический центр Национальной академии наук Беларуси по продовольствию», г. Минск, Республика Беларусь. Пищевая Промышленность: наука и технологии. 2017;1(35):63-68.

10. Бочаров В.А. Назарова Н.Е. Терехова А В. Мансуров А.П. Определение оптимальной концентрации кисломолочной закваски для ускорения ферментации и повышения качества квашеной капусты. Вестник Мичуринского государственного аграрного университета. 2016;(1):99-103.

11. Полянская И.С., Катаранов Г.О., Закрепина Е.Н. Подбор консорциума пробиотиков дпя продукта из молочной сыворотки. Пищевая индустрия. 2019:4(42):18-19. 12. Джей Дж.М., Лёсснер Дж., Гольден Д.А. Современная пищевая микробиология Пер. 7-го англ. изд. М.: БИНОМ. Лаборатория знаний, 2017. 886 с.

13. Комплексная программа развития биотехнологий в Российской Федерации на период до 2020 года Москва, 2012 // Электронные ресурсы: Технологическая платформа БИОТЕХ2030биоресурсы. http://biotech2030.ru/deyatelnost/nauchnotehnicheskoe-razvitie Дата доступа 01052020

14. Лютова Татьяна Викторовна Математическое моделирование ресурсного взаимодействия популяций микроорганизмов биотехнологических систем в условиях контаминации Автореферат диссертации на соискание ученой степени кандидата технических наук Воронеж, 2011 (10 фев 2011) (Электронный ресурс https:/dlib.rsl.ru (дата обращения 1601 2019))

15. Посокина Н.Е., канд. техн. наук, Лялина О.Ю. Деструкция фруктозы в процессе направленного фрерментирования огурцов с использованием штаммов молочнокислых микрооганизмов. Инновационные исследования и разработки для научного обеспечения производства и хранения экологически безопасной сельскохозяйственной и пищевой продукции: сборник материалов II Международной научно-практической конференции (05 - 26 июня 2017 г., г. Краснодар) Краснодар, 2017. с. 589 , 62-66

16. Соловьева А.Е., Шеленга Т.В., Артемьева А.М. Метаболомный подход к комплексной биохимической характеристике вида капуста огородная Brassica oleracea L. Овощи России. 2019;(4):72-79. https://doi.org/10.18619/2072-9146-2019-4-72-79

17. R. Cvetkovic $a$, Lato L. Pezob, Tatjana Tasic a, Ljubiša Šaric $a, Z^{\prime}$ arko Kevrešana Jasna Mastilovic. The optimisation of traditional fermentation process of white cabbage (in relation to biogenic amines and polyamines content andmicrobiological profile Biljana. Food chem. 2015;(168):471-477.

18. Jagannath A., Raju P.S. , Bawaa A.S. Two-step controlled lactic fermentation of cabbage for improved chemical and microbiological qualities. J.Food Quality. 2012:35(10):13-20.

19. Michelle A. Zabat, William H. Sano, Jenna I. Wurster ID, Damien J. Cabrall Dand Peter Belenky. Microbial Community Analysis of Sauerkraut Fermentation Reveals a Stable and Rapidly Established Community. Foods. 2018;(7):77. doi:10.3390/foods 7050077

20. Паспорт на штамм Leuconostoc mesenteroides subsp mesenteroides $37 Y$ ? выданный Всероссийской Коллекцией Промышленных Микроорганизмов ФГУП ГосНИИ Генетика, дата выдачи 01122016

21. Паспорт на штамм Lactobacillus brevis B-1309, получен во Всероссийском начно-исспедовательском институте пищевой биотехнологии - филиале Федерального государственного бюджетного учреждения науки Федерального исследовательского центра питания, биотехнологии и безопасности пищи, дата выдачи 28052019

22. Паспорт на штамм Lactobacillus plantarum 578/26, полученный во Всероссийском научно-исспедовательском институте пищевой биотехнологии филиале ФГБУН «ФИЦ питания и биотехнологии», дата выдачи 08042019.

23. ГOCT ISO 7218-2015 Микробиология пищевых продуктов и кормов для живот ных. Общие требования и рекомендации по микробиологическим исспедованиям.

\section{About the authors:}

Jeanne A. Semenova - Researcher

Nataliya E. Posokina - Cand. Sci. (Engineering), Head of the laboratory,

https://orcid.org/0000-0002-7857-6785

Vladimir I. Tereshonok - Cand. Sci. (Agriculture),

Senior Researcher

\section{- References}

1. Pozdnyakova O.G., Kazakova M.A., Austrievskikh A.N., Poznyakovsky V.M. Micronutrient support of a young organism in the conditions of urbanization of modern society. Agroindustrial complex of Russia. 2019;26(3):436-441. Chelyabinsk, ISSN 2587 8824. (In Russ.)

2. Hutkins R.W. Microbiology and Technology of Fermented Foods. Blackwell Publishing 2006

3. Shishlova E.S., Posokina N.E., Lyalina O.Yu. Basics of fermentation of white cabbage. Vestnik VSUIT. 2018;80(2):242-248. (In Russ.) doi: 10.20914 / 2310-1202-2018-2-242 248

4. Ganina V.I., Koroleva NS, Filchakova S.A. Technical Microbiology of Animal Products: Textbook. M.: DeLi print, 2008. 352 p. (In Russ.)

5. Vyschepan A.G., Melman M.E. Physicochemical bases of pickling and pickling vegetables. Moscow: Gostorgizdat, 1952. 158 p. (In Russ.)

6. Tursunbaeva G.N., Kasenova A.A., Chukanov N.K., Mukasheva G.M., Murzasheva N., Rakhimberlina R.M. The bacterial strain Lactobacillus brevis used in sauerkraut. Type: copyright certificate. Certificate number: SU 1622391 A1 Patent office: USSR, 1991. Application number: 4474295. Date of registration: 17.08.1988. Date of publication: 01/23/1991. Applicant: Institute of Microbiology and Virology of the Academy of Sciences of the KAZSSR (Electronic resource: Elibrary: access date 0804 2020). (In Russ.)

7. Shlykova A.N., Ustimova E.A., Belokurova E.S. Using starter cultures of microorganisms Lactobacillus plantarum 8p-a3 and Lactobacillus fermentum 39 in the production of sauerkraut. Proceedings of the conference Science Week SPBPU St. Petersburg, November 19-24, 2018, pp.47-50. (In Russ.)

8. Belokurova E.S., Ivanchenko O.B. Biotechnology of products of plant origin: a tutorial. SPb .: Publishing house "Lan", 2019. 232 p. (Textbooks for universities. Special literature). (In Russ.)

9. Pavlovskaya L.M., Golubeva S.N. Promising directions of scientific research of the processes of preserving vegetables and fruits: production of fermented products. RUE "Scientific and Practical Center of the National Academy of Sciences of Belarus for Food", Minsk, Republic of Belarus. Food Industry: Science and Technology. 2017;1(35):63-68 (In Russ.)

10. Bocharov V.A., Nazarova N.E., Terekhova A.V., Mansurov A.P. Determination of the optimal concentration of fermented milk starter culture to accelerate fermentation and improve the quality of sauerkraut. Bulletin of Michurinsk State Agrarian University 2016;(1):99-103. (In Russ.)

11. Polyanskaya I.S., Kataranov G.O., Zakrepina E.N. Selection of a consortium of probiotics for a whey product. Food industry. 2019;4(42):18-19. (In Russ.)

12. Jay J.M., Lessner J., Golden D.A. Modern food microbiology. Per. 7th eng. ed. M BINOM. Knowledge Laboratory, 2017. 886 p. (In Russ.)

13. Comprehensive program for the development of biotechnology in the Russian Federation for the period up to 2020 Moscow, 2012 // Electronic resources: Technological platform BIOTECH2030 bioresources. http://biotech2030.ru/deyatelnost/nauchnotehnicheskoe-razvitie Accessed 0105 2020. (In Russ.)

14. Lyutova Tatyana Viktorovna Mathematical modeling of the resource interaction of populations of microorganisms of biotechnological systems under conditions of contamination Abstract of the thesis for the degree of candidate of technical sciences Voronezh 2011 (10 Feb 2011) (Electronic resource: https: /dlib.rsl.ru (date of access 1601 2019)) (In Russ.)

15. Posokina N.E., Cand. tech. Sci., Lyalina O.Yu. Destruction of fructose in the process of directed fermentation of cucumbers using strains of lactic acid microorganisms. Innovative research and development for scientific support of production and storage of ecologically safe agricultural and food products: collection of materials of the II International Scientific and Practical Conference (05 - 26 June 2017, Krasnodar). Krasnodar, 2017, p. 589, 62-66. (In Russ.)

16. Solovyeva A.E., Shelenga T.V., Artemyeva A.M. The metabolomic approach to the complex biochemical characteristics of cole Brassica oleracea L. Vegetable crops of Russia. 2019;(4):72-79. (In Russ.) https://doi.org/10.18619/2072-9146-2019-4-72-79

17. R. Cvetkovic , Lato L. Pezob, Tatjana Tasic a, Ljubiša Šaric a, Žarko Kevrešana, Jasna Mastilovic. The optimisation of traditional fermentation process of white cabbage (in relation to biogenic amines and polyamines content andmicrobiological profile Biljana. Food chem. 2015;(168):471-477.

18. Jagannath A., Raju P.S. , Bawaa A.S. Two-step controlled lactic fermentation of cabbage for improved chemical and microbiological qualities. J.Food Quality. 2012:35(10):13-20.

19. Michelle A. Zabat, William H. Sano, Jenna I. Wurster ID, Damien J. Cabrall Dand Peter Belenky. Microbial Community Analysis of Sauerkraut Fermentation Reveals a Stable and Rapidly Established Community. Foods. 2018;(7):77. doi:10.3390/foods7050077

20. Passport for the strain Leuconostoc mesenteroides subsp mesenteroides 37Y? issued by the All-Russian Collection of Industrial Microorganisms FSUE GosNII Genetics, date of issue 0112 2016. (In Russ.)

21. Passport for the strain Lactobacillus brevis B-1309, obtained from the All-Russian Initial Research Institute of Food Biotechnology - a branch of the Federal State Budgetary Institution of Science of the Federal Research Center for Nutrition, Biotechnology and Food Safety, date of issue 2805 2019. (In Russ.)

22. Passport for the strain Lactobacillus plantarum 578/26, obtained at the All-Russian Research Institute of Food Biotechnology - branch of the Federal State Budgetary Scientific Institution "Federal Research Center of Nutrition and Biotechnology", date of issue 0804 2019. (In Russ.)

23. GOST ISO 7218-2015 Microbiology of food and animal feed. General requirements and recommendations for microbiological research. (In Russ.) 